\title{
Do We Really Need to Repair the Pronator Quadratus in Volar Plating of Distal Radius Fracture『A Protocol for Systematic Review and Meta Analysis
}

\section{Qiujiang Li}

Ningxia Medical University

\section{Xingxia Long}

Sichuan University West China Hospital

\section{Yinbin Wang}

People's Hospital of Ningxia Hui Autonomous Region

\section{Zhaofu Wang}

People's Hospital of Ningxia Hui Autonomous Region

\section{Donggeng Guo}

People's Hospital of Ningxia Hui Autonomous Region

Jinhan Lv

People's Hospital of Ningxia Hui Autonomous Region

\section{Feng Ma}

People's Hospital of Ningxia Hui Autonomous Region

\section{Lijun Cai ( $\nabla$ scslqj@163.com )}

Zhengyuan Street, Yinchuan, Ningxia

\section{Protocol}

Keywords: distal radius fracture, systematic review protocol, pronator quadratus

Posted Date: May 18th, 2021

DOI: https://doi.org/10.21203/rs.3.rs-521342/v1

License: (c) (1) This work is licensed under a Creative Commons Attribution 4.0 International License. Read Full License 


\section{Abstract}

\section{Introduction}

Distal radius fracture is a common type of fracture in clinical practice.Over recent years, Volar locking plate internal fixation remains the most commonly used surgical treatment method in the treatment of distal radius fractures. To better expose the distal radius and fracture segment, the surgeons have to cut the pronator quadratus $(P Q)$ muscle.However, whether the $P Q$ muscle need to be repaired remains controversial. This study aims to provide a plan to evaluate the clinical efficacy whether to repair the PQ muscle in volar plate fixation of distal radius fractures.

\section{Methods and analysis}

We will search PubMed, Cochrane Library, EMBASE, China National Knowledge Infrastructure (CNKI), and Wanfang databases from the beginning to February 2021 to compare clinical outcomes whether to repair the PQ muscle. Quality assessment will be evaluated using the risk of bias tool recommended by the Cochrane Handbook 5.2.0. Subsequently, Revman software version 5.3 will be used for data analysis when a meta-analysis is allowed including subgroup and sensitivity analysis.

\section{PROSPERO registration number CRD42020206959}

\section{Highlights}

This study will systematically evaluate the clinical efficacy,and applicability of repairing and nonrepairing the $P Q$ muscle in volar plate fixation of distal radius fractures.

- The research results will provide a reference for clinicians to choose an appropriate surgery for distal radius fractures.

- This study provides improved clinical applicability and comparability of derived results through subgroup analysis.

- The results of this study may change and need to be updated when new high-quality controlled trials are reported.

- We only include English and Chinese literatures, which may lead to a selection bias.

\section{Introduction}

Distal radius fractures are responsible for high incidence worldwide, mostly due to the anatomy of distal radius,which is located between cortical and cancellous bone ${ }^{[1,2]}$.According to relevant research statistics, distal radius fractures are the most common upper limb fractures ${ }^{[3]}$. Currently, open reduction and volar plate internal fixation of distal radius fractures is the best treatment option ${ }^{[4-6]}$.To better place 
on the deep surface of the PQ muscle, it is necessary to be dissected during the operation ${ }^{[7-9]}$.However, whether to repair the $P Q$ muscle remains controversial.83\% (608/753) members of the American Society of Hand Surgery of a previous study recommended that they tried to repair the PQ muscle ${ }^{[10]}$. The main reason is that it is more beneficial to the recovery of the $P Q$ strength, the stability of the distal radioulnar joint, and avoid the damage caused by the long-term friction between the volar flexor tendon and the plate. Another part of the researchs believe that repairing the $P Q$ muscle will further aggravate the ischemic contracture of the muscle, which will eventually affect the range of motion of the forearm ${ }^{[11]}$.Two studys published in 2013 found that there is no correlation between the repairing of the $P Q$ muscle and the postoperative efficacy and functional recovery of the distal radius fractures ${ }^{[12,13]}$. The results of another study showed that repairing the $P Q$ muscle can reduce the short-term pain of postoperative patients ${ }^{[14]}$. Many experiments have compared the effect of repairing and not repairing the $P Q$ muscle on the postoperative efficacy of distal radius fractures ${ }^{[7,12-14]}$. However, there is still a lack of high-quality systematic reviews. Therefore, this study uses a meta-analysis to systematically evaluate and compare the curative effect and applicability of repairing and not repairing the PQ muscle, so as to provide a basis for orthopedic trauma surgeons to choose appropriate surgical methods.

\section{Methods}

\subsection{Inclusion criteria}

\subsubsection{Type of study}

randomized controlled trials (RCTs) and comparative experimental trials will be included in this SR. Patients should be randomly assigned into with $P Q$ repair and without $P Q$ repair groups. The language of the literature will be limited in English or Chinese.

\subsubsection{Participants}

All patients were clearly diagnosed as fresh closed distal radius fractures by X-ray or CT. Patients with metachronous fractures, open fractures, or combined with vascular and nerve injury were excluded.

\subsubsection{Interventions}

Under brachial plexus anesthesia or general anesthesia, the patient is in a supine position, and the radial styloid process is taken from the transverse carpal striae, and an incision extending about $8 \mathrm{~cm}$ proximally is exposed from the approach between the flexor carpi radialis muscle and the radial artery and vein. The lateral flexor carpi tendon is pulled to the ulnar side, and the radial artery and vein are properly pulled to the radial side. Properly bluntly separated along the direction of the muscle fibers to expose the distal and proximal ends of the $P Q$ muscle covering the distal radius, and run longitudinally in $1 / 3$ of the radial side ${ }^{[9,13]}$. The $P Q$ muscle was incised to expose the fractured radius for reduction and internal fixation with plate screws. After the internal fixation operation is completed, the incision is flushed with saline. In group A, the incised PQ muscle was sutured carefully with absorbable sutures, and 
then the skin was sutured layer by layer, while in group B, the incised PQ muscle was stretched to cover the steel plate and the tissue was directly sutured layer by layer ${ }^{[12-14]}$.

Postoperative treatment generally does not require plaster fixation. After 24 , the extension and flexion function exercises of the affected metacarpophalangeal joint and interphalangeal joint will be started. After 2 days, postoperative X-ray photography will be performed to check the fracture reduction and internal fixation ${ }^{[7,12]}$.

\subsubsection{Outcomes}

The primary outcomes will include the wrist pain intensity measurement via a Visual Analogue Scale (VAS) or a Numerical Rating Scale (NRS) within 2 weeks after surgery.

The range of motion (ROM) of the wrist on the affected side and the disabilities of the Arm, Shoulder and Hand (DASH) score will be collected within 1year after surgery.Secondary results include operation time, intraoperative blood loss, and adverse events (vascular and nerve damage and compartment syndrome).

\subsection{Date sources}

We will search the following databases from establishment to February 2021: PubMed, Cochrane Library, EMBASE, China National Knowledge Infrastructure (CNKI), and Wanfang databases from the beginning to February 2021.

\subsection{Serarch strategy}

Search strategy will follow the Cochrane handbook. The search strategy for PubMed is shownin Table 1, and similar strategies will be built and applied forother electronic databases. The search strategy is listed in Table 1.

Table 1 search strategy used in PubMed database

\begin{tabular}{|ll|}
\hline Number & Search termse \\
\hline$\# 1$ & distal radi*[Title/Abstract] \\
\hline$\# 2$ & volar plat*[Title/Abstract] \\
\hline$\# 3$ & pronator quadratus[Title/Abstract] \\
\hline$\# 4$ & $\# 1$ AND \#2 AND \#3 \\
\hline
\end{tabular}

\subsection{Study selection and data extraction}

After selecting the study based on the inclusion criteria according to the abovementioned,two independent reviewers will read the full texts of each article to extract pertinent data using a data extraction form. Any disagreements will be solved by discussion 
or consultation with another researcher. If the primary data is missing,incomplete,or reported in the form of graphs,we will contact the author by email or phone calls for raw data. If the author of the paper has lost the relevant data,or does not agree to provide data,we will give a statistical description in the results section instead of meta-analysis. The research summary of the screening flow chart is shown in Fig. 1.

\subsection{Quality assessment of included studies}

Two independent assessment of risk of bias researchers will individually use the risk of bias (RoB) tool proposed by Cochrane Handbook V.5.2.0 to assess the quality risk of bias (RoB) for randomized controlled trials (RCTs), which included six aspects: (1) Random sequence generation, (2) Allocation concealment, (3) Blinding of patients and personnel, (4) Incomplete outcome data, (5) Selective reporting, (6) Other bias. The RoB in each field is divided into "Low risk," "High risk," or "Unclear risk." . If the RoB of the research included in the meta-analysis is different, we will conduct a hierarchical analysis based on the RoB to show readers different results under different RoBs.

\subsection{Subgroup analysis}

If heterogeneity is detected, a subgroup analysis will be performed to explore differences in methodological quality, age, and AO fracture classification.

\subsection{Data synthesis and analysis}

The meta-analysis in this review will use RevMan 5.3 and Stata 13.0 software. For the outcome index of two categorical variables, relative risk will be used, and for the outcome index of continuous variables, the average difference or standardized average difference will be used, with a confidence interval of $95 \%$. The heterogeneity test will be used for the included studies, and these studies will be tested by the Higgins ${ }^{2}$ test. If there is a low heterogeneity $\left(I^{2} \leq 25 \%\right)$, a fixed-effect model will be used;otherwise, a random effects model $\left(I^{2}>25 \%\right)$ will be used ${ }^{[15]}$. If the $I^{2}$ value of the combined results is greater than $75 \%$, we will abandon the meta-analysis and only give a general statistical description of the results ${ }^{[15]}$.Further analyze the source of heterogeneity and, if necessary, perform subgroup analysis. There are clinical and methodological differences in experimental research. Therefore, this study will choose the random effects model. Finally, a funnel chart will be drawn to assess the publication bias of the literature. If enough research is included, sensitivity analysis will be performed to test the robustness of the results. We will conduct a sensitivity analysis by exclusion. Studies with high risk of bias and outliers that are numerically far away from the rest of the data.

\subsection{Publication deviation}

If the results of the meta-analysis include more than 10 articles, we will use a funnel chart to test whether there is publication bias. If the number of articles included in the study is less than 10 , the publication bias is not significant.

\subsection{Quality of evidence}


The quality evidence of the included studies will use the Grading of Recommendation, Assessment, Development and Evaluation (GRADE) approach, classified evidence as high, moderate, low or very low quality based on considerations of RoB, consistency, directness, precision and publication bias ${ }^{[16]}$.

\subsection{Patient and public involvement}

Patients and the public were not involved in the design or planning of systematic review protocol.

\section{Discussion}

A fracture of the distal radius refers to a fracture within $3 \mathrm{~cm}$ of the articular surface of the distal radius ${ }^{[17]}$. With the aging of the population, the incidence of distal radius fractures in elderly patients is increasing ${ }^{[1,4]}$. The PQ muscle is close to a quadrilateral and is divided into a deep head and a shallow head $^{[18]}$. The shallow head plays a major role in the forearm pronation process, the deep head plays a major role in the stability of the distal radioulnar joint, and the deep and shallow head work together to maintain the pronation function of the forearm ${ }^{[19]}$. Therefore, repairing the PQ muscle should help the functional recovery of patients after internal fixation of distal radius fractures. However, some clinical studies compared cases with and without $P Q$ repair after the volar plate of the distal radius, and did not show a significant difference in pronation strength. Therefore, there is controversy about repairing or not repairing the $P Q$ muscle. Unfortunately, there is a lack of high-quality systematic reviews or meta-analysis publications, and no systematic and scientific evaluation of them.

We will conduct this research strictly in accordance with the Cochrane Systematic Review Manual, and report in accordance with the preferred reporting items of the guidelines for systematic review and metaAnalysis. We hope to evaluate and compare the with repaire and without repaire the PQ muscles through a systematic review, and provide some suggestions for its reasonable and effective clinical application.

\section{TRIAL STATUS}

- Preliminary searches: started.

- Piloting of the study selection process: started.

- Formal screening: started.

- Data extraction: started.

- RoB assessment: not started.

Data analysis: not started

\section{Abbreviations}


$\mathrm{PQ}=$ pronator quadratus, $\mathrm{RCTs}=$ randomized controlled trials, VAS=Visual Analogue Scale, NRS= Numerical Rating Scale, ROM=range of motion, DASH=disabilities of the Arm, Shoulder and Hand, GRADE=Grading of Recommendation, Assessment, Development and Evaluation, RoB=risk of bias.

\section{Declarations}

\section{Ethics and dissemination}

Given the nature of this study, no ethical approval will be required. The protocol will be disseminated via a peer-reviewed journal.

\section{Disclosure}

The authors have no personal, financial, or institutional interests in any of the drugs, materials, or devices described in this article.

\section{Funding}

This study was funded by Ningxia Hui Autonomous Region Science and Technology Benefiting People Special Project(2018KJHM00) .

\section{Availability of data and materials}

Please contact the corresponding author for data requests.

\section{Author details}

${ }^{1}$ Graduate School of Ningxia Medical University, Yinchuan, Ningxia, China.

${ }^{2}$ Department of Orthopedics,People's Hospital of Ningxia Hui Autonomous Region, Yinchuan, Ningxia, China.

${ }^{3}$ West China Hospital, Sichuan University,Sichuan,China

*Correspondence: Lijun Cai, No. 56, Zhengyuan Street, Yinchuan, Ningxia, 750002, China.(email:scslqj@163.com)

\section{Author contributions}

Conceptualization: Lijun Cai.

Data curation:Qiujiang Li, Xingxia Long, Yinbin Wang.

Formal analysis: Qiujiang Li.

Funding acquisition: Donggeng Guo, Jinhan Lv, Lijun Cai. 
Project administration: Feng Ma, Lijun Cai.

Resources: Feng Ma, Lijun Cai.

Writing - original draft: Qiujiang Li, Xingxia Long, Yinbin Wang, Zhaofu Wang.

Writing - review \& editing: Qiujiang Li, Xingxia Long.

\section{References}

1. Corsino CB, Reeves RA, Sieg RN. Distal Radius Fractures[J]. 2021.

2. Levin LS, Rozell JC, Pulos N. Distal Radius Fractures in the Elderly[J]. J Am Acad Orthop Surg,2017,25(3):179-187.

3. Mauck BM, Swigler CW. Evidence-Based Review of Distal Radius Fractures[J]. Orthop Clin North Am,2018,49(2):211-222.

4. Luokkala T, Laitinen MK, Hevonkorpi TP, et al. Distal radius fractures in the elderly population[J]. EFORT Open Rev,2020,5(6):361-370.

5. Ermutlu C, Mert M, Kovalak E, et al. Management of Distal Radius Fractures: Comparison of Three Methods[J]. Cureus,2020,12(8):e9875.

6. Saving J, Ponzer S, Enocson A, et al. Distal radius fractures-Regional variation in treatment regimens[J]. Plos One,2018,13(11):e0207702.

7. Zhang J, Zhuang YQ, Zhou L, et al. Sparing the pronator quadratus for volar plating of distal radius fractures: a comparative study of two methods[J]. J Int Med Res. 2020;48(8):300060519893851.

8. Liverneaux PA. The minimally invasive approach for distal radius fractures and malunions[J]. J Hand Surg Eur Vol,2018,43(2):121-130.

9. Hohendorff B, Knappwerth C, Franke J, et al. Pronator quadratus repair with a part of the brachioradialis muscle insertion in volar plate fixation of distal radius fractures: a prospective randomised trial[J]. Arch Orthop Trauma Surg,2018,138(10):1479-1485.

10. Swigart CR, Badon MA, Bruegel VL, et al. Assessment of pronator quadratus repair integrity following volar plate fixation for distal radius fractures: a prospective clinical cohort study[J]. J Hand Surg Am. 2012;37(9):1868-73.

11. Mcconkey MO, Schwab TD, Travlos A, et al. Quantification of pronator quadratus contribution to isometric pronation torque of the forearm[J]. J Hand Surg Am,2009,34(9):1612-7.

12. Hershman SH, Immerman I, Bechtel C, et al. The effects of pronator quadratus repair on outcomes after volar plating of distal radius fractures[J]. J Orthop Trauma. 2013;27(3):130-3.

13. Tosti R, llyas AM. Prospective evaluation of pronator quadratus repair following volar plate fixation of distal radius fractures[J]. J Hand Surg Am,2013,38(9):1678-84.

14. Haberle S, Sandmann GH, Deiler S, et al. Pronator quadratus repair after volar plating of distal radius fractures or not? Results of a prospective randomized trial[J]. Eur J Med Res. 2015;20:93. 
15. Higgins JP, Thompson SG, Deeks JJ, et al. Measuring inconsistency in meta-analyses[J]. BMJ,2003,327(7414):557 - 60.

16. Puhan MA, Schunemann HJ, Murad MH, et al. A GRADE Working Group approach for rating the quality of treatment effect estimates from network meta-analysis[J]. BMJ,2014,349:g5630.

17. Yin SQ, Huang YP, Li MZ, et al. [Relationship between radiographic parameters and clinical outcomes of elderly patients with distal radius fractures][J]. Zhongguo Gu Shang. 2018;31(2):141-4.

18. Carlson TL, Bhandari L, Chang J, et al. Pronator quadratus muscle flap: a preliminary cadaveric study[J]. Eur J Orthop Surg Traumatol,2020,30(1):103-107.

19. Fang K, Lin X, Liu X, et al. Do we need to suture the pronator quadratus muscle when we do open reduction and internal fixation for fracture of the distal radius[J]. BMC Musculoskelet Disord. 2020;21(1):453.

\section{Figures}



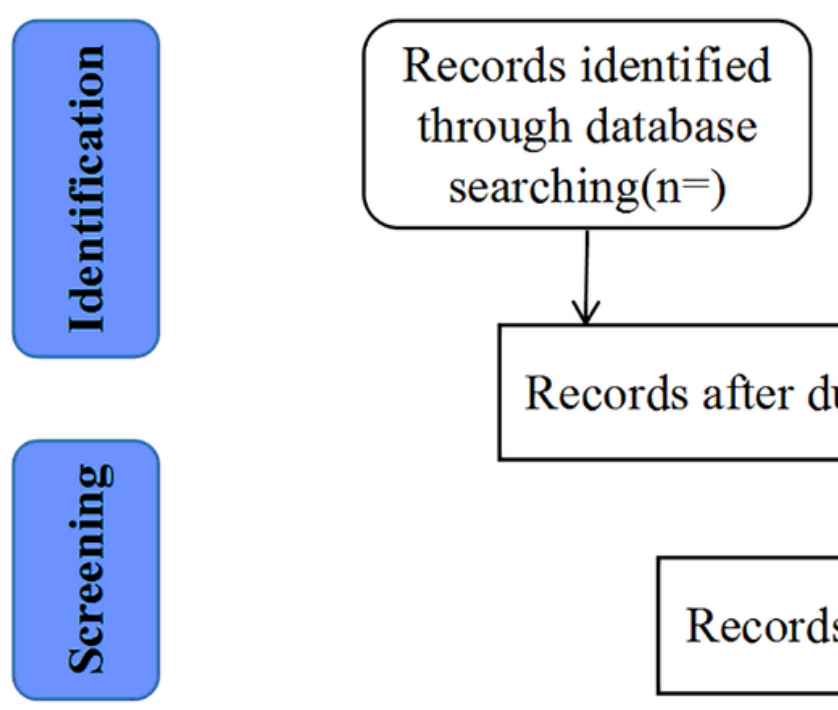

Additional records identified through other $\operatorname{sources}(\mathrm{n}=)$

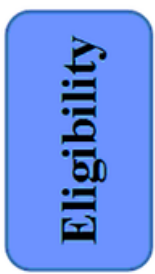

Records after duplicates removed( $\mathrm{n}=$ )
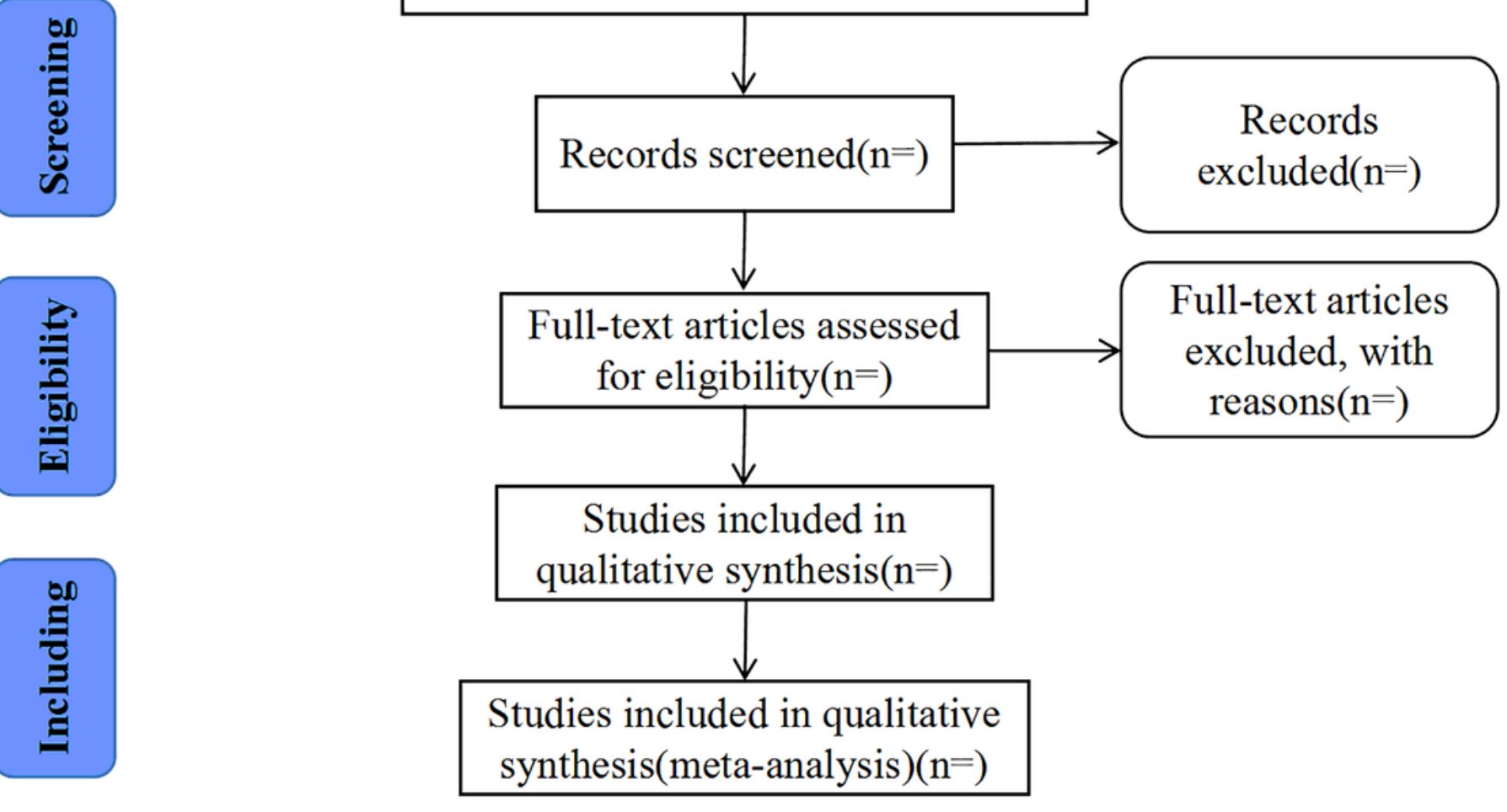

Figure 1

Flow diagram of studies identified.

\section{Supplementary Files}

This is a list of supplementary files associated with this preprint. Click to download.

- PRISMA2009ChecklistMSWord.doc 